\title{
Retinal Blood Vessel Extraction from Fundus Images Using Enhancement Filtering and Clustering
}

\author{
Jyotiprava Dash1* ${ }^{*}$ Priyadarsan Parida2 ${ }^{+}$and Nilamani Bhoi3 ${ }^{+}$ \\ * Department of Electronics and Telecommunication Engineering, Veer Surendra Sai University of Technology, Burla, \\ Sambalpur, India \\ + Department of Electronics and Communication Engineering, GIET University, Gunupur, Rayagada, India \\ + Department of Electronics and Telecommunication Engineering, Veer Surendra Sai University of Technology, Burla, \\ Sambalpur, India
}

Received 25 May 2020; accepted 14 July 2020

\begin{abstract}
Screening of vision troubling eye diseases by segmenting fundus images eases the danger of loss of sight of people. Computer assisted analysis can play an important role in the forthcoming health care system universally. Therefore, this paper presents a clustering based method for extraction of retinal vasculature from ophthalmoscope images. The method starts with image enhancement by contrast limited adaptive histogram equalization (CLAHE) from which feature extraction is accomplished using Gabor filter followed by enhancement of extracted features with Hessian based enhancement filters. It then extracts the vessels using Kmean clustering technique. Finally, the method ends with the application of a morphological cleaning operation to get the ultimate vessel segmented image. The performance of the proposed method is evaluated by taking two different publicly available Digital retinal images for vessel extraction (DRIVE) and Child heart and health study in England (CHASE_DB1) databases using nine different performance matrices. It gives average accuracies of 0.952 and 0.951 for DRIVE and CHASE_DB1 databases, respectively.
\end{abstract}

Key Words: Fundus images, retinal vasculature, morphological cleaning, K-mean clustering.

\section{Introduction}

Eye is the very distinctive region of the human body where the vascular condition can be directly observed in vivo. Retinal blood vessels constitute the vital theme of screening schemes and play a significant role in instinctive retinal ailment finding [1], [2]. Exact extraction of fundus vasculature could be enable the estimation of choroidal neovascularization, retinal artery occlusion, retinopathy prematurity, cardiovascular diseases, hypertension etc. Fundus image segmentation approaches are based on the contrast among the blood vessels and its background. Manual blood vessel segmentation is a complex task as it requires training. So, in the present time automatic techniques are developing which can save time, cost and patient vision effectively. In the literature, many methods are developed for vasculature extraction, it can categorize which as supervised and unsupervised technique of segmentation.

Correspondence to: priyadarsan.vssut@gmail.com

Recommended for acceptance by Angel D. Sappa

https://doi.org/10.5565/rev/elcvia.1239

ELCVIA ISSN: 1577-5097

Published by Computer Vision Center / Universitat Autonoma de Barcelona, Barcelona, Spain 
Supervised techniques are reliant on sorting of pixels where each pixel is categorized in to two sets such as vessels and non-vessels. Here classifiers are trained by supervised learning with data from hand labeled images [3]. In [3], Soares et al. used Gabor filter and Bayesian classifier for extraction of blood vessels from fundus images. In [4], You et al. used a combination of radial projection and modified steerable complex wavelet to extract the retinal blood vessels. In [5], the author offered a method that segments the blood vessels using 7D feature vector and neural network. In [6], a supervised algorithm have been offered by Fraz et al. for blood vessel extraction using a collective classifier of improved and bagged decision trees. In [7], the author proposed a lattice neural network with dendritic processing based method for retinal blood vessel extraction. In [8], Roychowdhury et al. proposed a novel segmentation method to extract blood vessels where the first stage extract the major vessels from the common region of binary images produced by the high pass filtering and morphological reconstruction. Then Gaussian mixture model (GMM) is used to classify vessel and non-vessel pixel. Finally, major vessels are combined with the identified vessel pixel to give the final segmentation output. In [9], Tan et al. extracted the retinal vasculature using convolutional neural network. In [10], the author used 7 layer convolutional neural network to segment the retinal blood vessel. In [11], Aslani et al. introduced a hybrid method using multi-scale feature analysis and ensemble RF classifier to extract the retinal blood vessels.

Unsupervised methods extracts the blood vessels by searching intuitive patterns of the vessels and without using classifier training phase during segmentation [12]. In [1], Mendonca et al. proposed a retinal vasculature extraction method that combines differential filters and morphological operators for centerline detection and gap filling in segmented vessel. In [2], the author derived an algorithm that initially design a bank of 180 Gabor filter followed by an imperialism competitive algorithm for optimizing the Gabor filter parameters. Finally, threshold values are determined to get final vessel segmented image. In [13], the author proposes a level set and region growing based method for blood vessel segmentation. In [14], Fraz et al. used a unique combination of vessel center line detection and morphological bit plane slicing to extract blood vessels from fundus images. In [15], the author elaborated an algorithm for the segmentation of blood vessel using multidirectional morphological bit plane slicing. In [16], Zhang et al. used matched filter with first order derivative of Gaussian to extract retinal vasculature. In [17], Cinsdikici et al. introduced a hybrid algorithm of matched filter and ant colony optimization to segment the retinal blood vessels from fundus images. In [18], the author proposed a genetic algorithm based technique that optimizes the parameters of matched filter to extract the blood vessels. In [19], Azzopardi et al. offered an unsupervised method that segments the blood vessels using bar selective COSFIRE filter. In [20], Budai et al. proposed a vessel extraction algorithm using Hessian matrix and hysteresis thresholding. In [21], Odstrcilik et al. presented a method that extract the blood vessel using kittler minimum error thresholding method. In [22], Mapayi et al. proposed a local adaptive thresholding method using gray level co-occurrence matrix-energy information to segment retinal blood vessels. In [23], Panda et al. used a novel binary Hausdorff symmetry measure based seeded region growing technique to segment retinal blood vessels. In [24], Roychowdhury et al. proposed an unsupervised method of blood vessel segmentation that initially segment the major vessel and then add finer vessel branches by adaptive global thresholding method. In [25], the author extract retinal blood vessel from fundus images using ribbon of twin active contour model. In [26], the blood vessel segmentation process starts with filtration of retinal images using Gabor kernels followed by image thinning. The vessel segmented image is obtain in the later stage using Ramer-Douglas-Peucker algorithm. In [27], Chutatape et al. proposed an unsupervised method for blood vessel extraction by using a unique combination of second order derivative of Gaussian filter and Kalman filter. In [28], the author presented a multi-scale analysis based method using gradient magnitude and maximum principal curvature of Hessian tensor.

Even though, from the literature we can perceive that there exists different methods for retinal vasculature extraction. Accurate vessel extraction is still a challenging task as we can see that the extracted vessel may lose connectivity among pixels, may incur false detection of vessel points or may not perform better in detecting thin vessels. Therefore, in this paper an unsupervised method has been developed which not only overcome the above challenges but also takes very less time, simple and easier to implement as compared to many existing algorithms. The method starts with image quality improvement by contrast limited adaptive histogram equalization from which it performs feature extraction using Gabor filter followed by the 
heightening of extracted features with Hessian based enhancement filters. It then extracts the vessels using K-mean clustering technique. Finally, the technique halts with the application of a morphological cleaning operation to get the eventual vessel segmented image.

The rest of this paper is described as follows. Section 2 describes the proposed segmentation technique. Section 3 describes experimental evaluations and comparisons. At last Section 4 explains the conclusion part.

\section{Methodology}

For retinal blood vessel segmentation, in the present article we have presented an unsupervised method based on clustering technique. The proposed method pre-processed the extracted green channel from original RGB image using contrast limited adaptive histogram equalization, Gabor filter and Hessian based enhancement filter. It then passes the pre-processed image through the k-mean clustering phase to extract the vessels from background followed by a morphological cleaning step to get the final vessel segmented image. The Fig. 1 elaborates the entire segmentation process is elaborated with the help of a block diagram.

\section{PRE-PROCESSING}

Before going to the segmentation stage, the quality of image should be improved which is achieved through the pre-processing phase. In retinal imaging, we consider the green channel image to perform the segmentation process due to its maximum contrast while red channel is saturated and low contrast channel and blue channel exhibits poor dynamic range [29]. Fig. 2 (a) and Fig. 2 (b) shows the original RGB image and green channel image. Pre-processing step includes the following sub steps.

\section{Contrast limited adaptive histogram equalization (CLAHE)}

CLAHE is a developed form of the adaptive histogram equalization which distributes the image into small sections and works on a distinct area where the contrast of individual small area is intensified so that the histogram of the yield image links to the histogram stated by the distribution parameter. The minor neighboring units are then combined by bilinear interpolation that overcome the falsely induced limits [30]. Over noise intensification can be avoided by controlling the difference of the individual similar area. This is used to correspondingly allocate the intensity values of the image and makes the unseen structures more perceptible [31]. The Fig. 2(c) shows output of the CLAHE. 


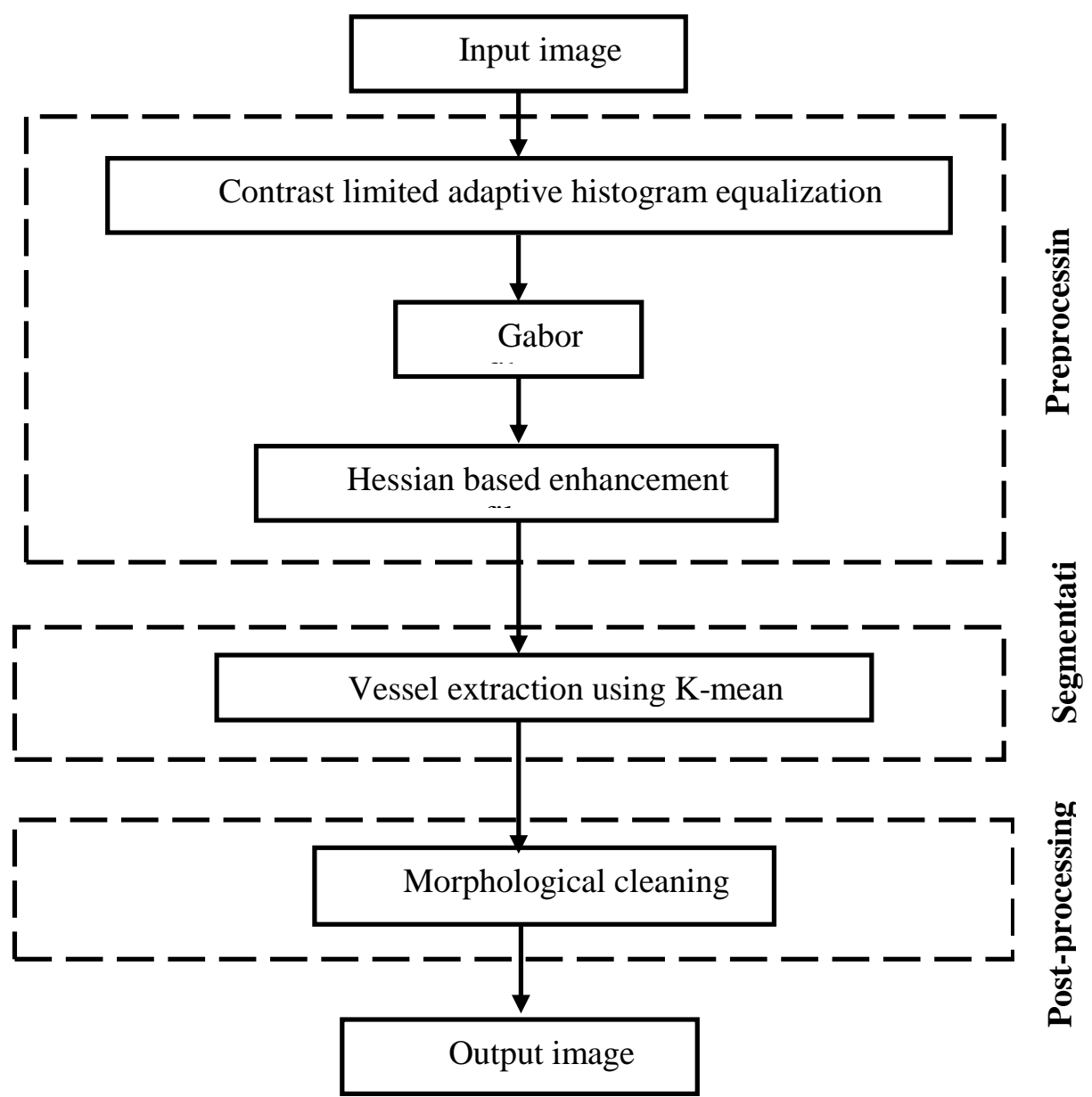

\section{Feature extraction using Gabor filter}

The proposed method uses Gabor filter to extract the features from the image obtained using CLAHE. The important characteristic of Gabor filter is its directional selectiveness capacity of unique oriented features and acceptable tuning to specific frequencies [32]. The Gabor filters are robust against intensity changes and noise. The Gabor filter based features are directly extracted from the CLAHE enhanced image. A 2D Gabor filter in spatial domain can be defined as a Gaussian kernel function modulated by a complex sinusoidal plane wave which can be given as,

$$
\begin{aligned}
& g(p, q)=\frac{F^{2}}{p i * a * y} \exp \left(-\frac{p^{2}+a^{2} q^{r^{2}}}{2 \sigma^{2}}\right) \exp \left(i * 2 * p i * F * p^{\prime}+\emptyset\right) \\
& p^{\prime}=p \cos \theta+q \sin \theta \\
& q^{\prime}=-p \sin \theta+q \cos \theta
\end{aligned}
$$

where, $F$ denotes the sinusoid frequency, $\theta$ angle of rotation of a Gabor function. $\phi$ is the phase offset, $\sigma$ is the standard deviation of the Gaussian envelope and $\alpha$ is the aspect ratio in spatial domain that specifies the ellipticity of the support of the Gabor function [33]. For obtaining highest performance accuracy the optimum value for the aspect ratio, frequency and angle of rotation are set to $4,0.6$ and $45^{\circ}$ respectively. The output is shown in Fig. 2 (d).

\section{Hessian based enhancement filter}

The Hessian-based enhancement filter method is based on the eigenvalue study of the Hessian matrix equivalent to the respective image part in two-dimensional images. The filter's response can be obtained by calculating and ordering the Hessian eigenvalues corresponding to the element [34]. 
Let $f(x)$ be the intensity function at $x$. The Hessian matrix $H(x)$ corresponding to the pixel at $x=(x, y)$ can be given as,

$H(x)=\left[\begin{array}{ll}\frac{\partial^{2} f}{\partial x^{2}} & \frac{\partial^{2} f}{\partial x \partial y} \\ \frac{\partial^{2} f}{\partial y \partial x} & \frac{\partial^{2} f}{\partial y^{2}}\end{array}\right] \triangleq\left[\begin{array}{ll}f_{x x} & f_{x y} \\ f_{y x} & f_{y y}\end{array}\right]$

For suitability and without causing uncertainty, let $\wedge=\left(\lambda_{1}, \lambda_{2}\right)$ with $\left|\lambda_{1}\right| \unrhd\left|\lambda_{2}\right|$ be the eigenvalues of Hessian matrix $H(x)$. The response function $R(x)$, of Hessian based enhancement filter can be expressed as,

$$
R(x)=\left\{\begin{array}{c}
R(\Lambda) \text {, if condition } \Omega_{\AA} \\
0, \text { otherwise }
\end{array}\right.
$$

The condition $\Omega_{\wedge}$ in the response function $R(x)$ in eq. (3) is linked with the Hessian eigenvalues. The condition $\Omega_{\wedge, b 2}$ and $\Omega_{\wedge, t 2}$ for identifying the bright structure like vessels can be defined as,

$$
\begin{aligned}
& \Omega_{\Lambda_{b} b 2}: \lambda_{1}<0, \lambda_{2}<0 \\
& \Omega_{\Lambda_{t} t 2}: \lambda_{1}<0
\end{aligned}
$$

Fig. 2 (e) represents the obtained enhanced image using Hessian based enhanced filter. The Hessian based enhanced filter makes the edges darker than background.

\section{VESSEL EXTRACTION USING K-MEAN CLUSTERING}

Clustering splits a set of data into a precise number of collections. Based on the features, K-mean clustering sets the objects into $\mathrm{K}$ groups where $\mathrm{K}$ is a positive integer. The $\mathrm{K}$-mean clustering is carried out in two phases where first phase computes the $\mathrm{K}$ centroid and second phase minimizes either each point to the group that has the closest centroid from the particular data point or the Euclidean distance between the data point. After the grouping phase, the new centroid is recalculated and according to this the Euclidian distance is calculated again and assigns the points in the cluster which have a minimum distance. So K-means is a recursive process in which it minimizes the sum of distances from respective object to its cluster centroid. The K-mean algorithm is given below [35].

Let $I_{H}(i, j)$ is the enhanced image of size $i \times j$, which have to be grouped into $\mathrm{K}$ number of clusters. Let $p(i, j)$ be the image pixel and $C_{K}$ is the cluster center.

1. Initialize the number of clusters and center.

2. Compute the Euclidean distance between center and each pixel is computed for every pixel of the image as, $D(i, j)=\left\|p(x, y)-C_{K}\right\|$.

3. Allot all the pixels to the adjacent center based on distance D.

4. After allotment of all pixels, recalculate the new position of the center as,

$$
c_{K}=\frac{1}{K} \sum_{j \in C_{K}} \sum_{i \in C_{K}} p(i, j)
$$

5. Repeat the above steps until it reaches the error value.

6. Reshape the cluster pixels into image.

The selection of cluster plays an important role in the performance of segmentation. So, to get better performance accuracy the number of clusters should be chosen carefully. In this method the number of clusters taken is 2 because here the pixels are classified as vessels or non-vessels.

\section{POST-PROCESSING}

After getting the output from the segmentation process, we can observe that the resulted image may contain some undesired pixel which may increase the false positives. So, to get only the desired vessels some further processing have to be accomplished which is achieved by morphological cleaning operation. The 
cleaning action removes all the pixels solely encircled by vessel points but not considered as a portion of a vessel. This is finished by seeing that each pixel with at least six neighbors marked as vessel points must also belong to a vessel [14], [36]. The Fig. 3 represents the result of K-mean clustering and post-processing. The Fig. 4 presents the segmentation output of DRIVE and CHASE_DB1 databases.

(a)

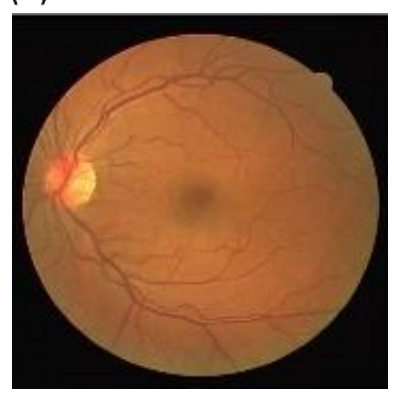

(d)

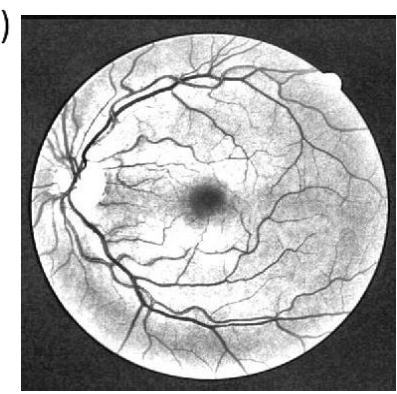

(b)

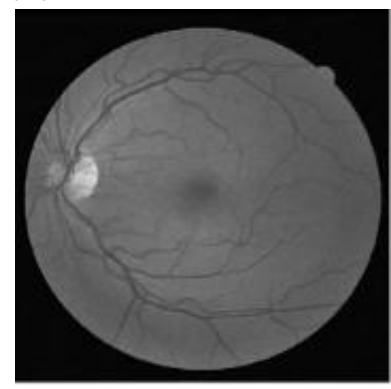

(c)

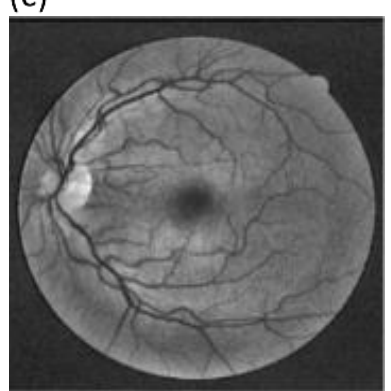

(e)

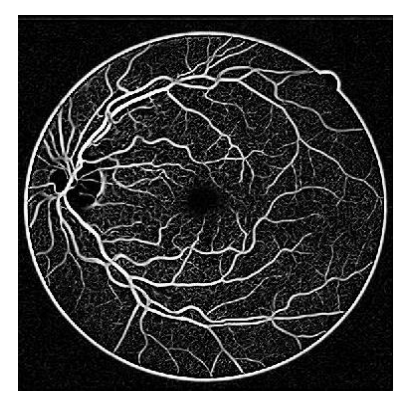

Fig.2. Output of pre-processing: (a) original image, (b) green channel extracted image, (c) CLAHE enhanced image, (d) Gabor feature extracted image and (e) Hessian based enhanced filtered image.

(a)
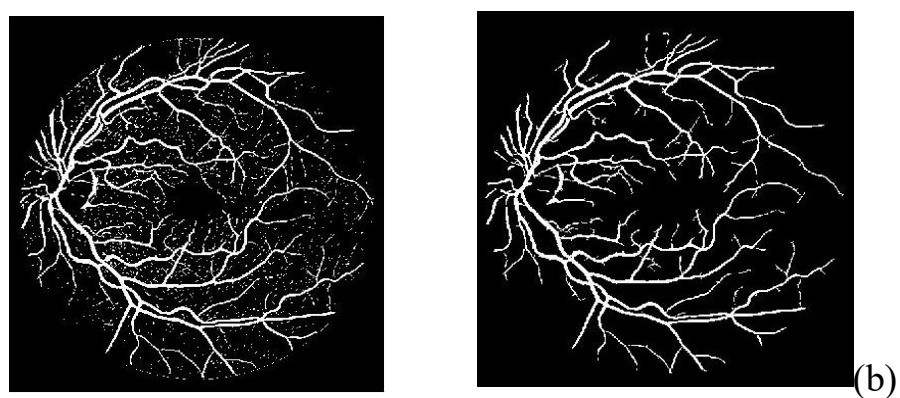

Fig.3. Output image: (a) before post-processing and (b) after post-processing 

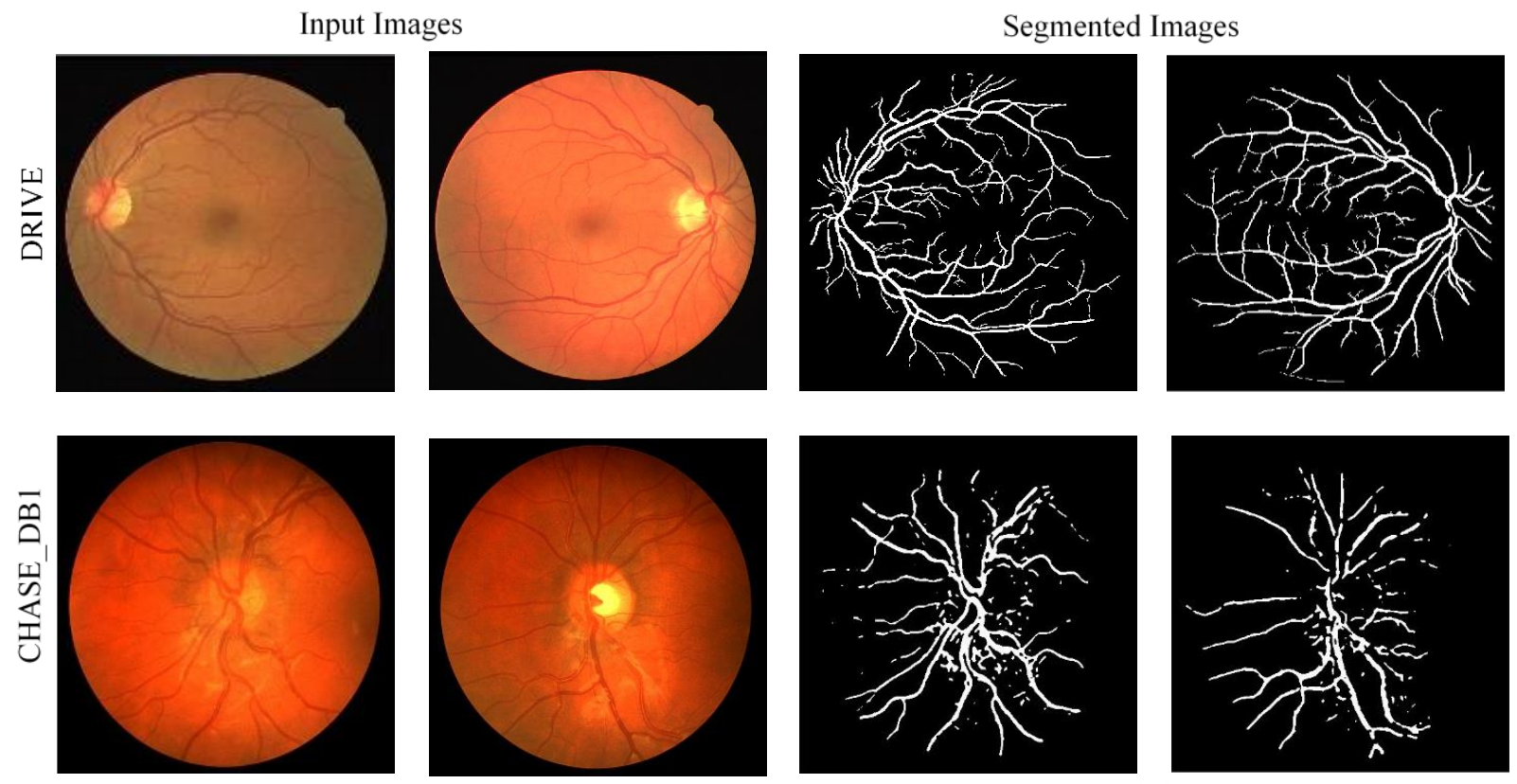

(a)

(b)

Fig. 4 Segmentation results for DRIVE and CHASE_DB1 databases (a) original images, (b) segmented images

\section{Results and discussions}

The performance of the proposed technique is evaluated and tested with the support of two widely available DRIVE (Digital retinal images for vessel extraction) and CHASE DB1 (Child heart and health study in England) databases. In DRIVE dataset [37], the pictures are captured with a canon CR5 nonmydriatic 3CCD camera with a $45^{\circ}$ field of view (FOV). The dataset contains 40 images which can be distributed into a training and a test set where apiece of them contains 20 images where 7 images comprise pathology. Image mask and ground truth images are available for all the images. The CHASE_DB1 [38][38] contains images of both the eyes of 28 children. It provides the manual segmentation results for the images.

The performance of the proposed technique is assessed by comparing the segmented image with the ground truth image. For creation of ground truth image, a vessel mask is created where vessel points are set to one and non-vessel points are set to zero. According to the pixel classification as vessel and non-vessel the four different possible combinations are true positive (TP), true negative (TN), false positive (FP) and false negative (FN) [39]. If the pixels are acknowledged as vessels in both the ground truth and resulted image then the grouping is called as TP. TN when the pixels are ordered as non-vessel in both ground truth and resulted image. When a pixel is recognized as non-vessel in resulted image but as vessel in ground truth image, it is termed as FN. The FP is defined as when a pixel is noticeable as vessel in resulted image and non-vessel in ground truth image. This paper used nine different performance matrices such as, of sensitivity (Se), specificity (Sp), positive predictive value (PPV), negative predictive value (NPV), false discovery rate (FDR), Matthews's correlation coefficient (MCC), Jaccard index (JI), dice coefficient (DC) and accuracy (ACC) for examination of performance. The Table 1 represents the mathematical formulation for the nine performance matrices. The proportion of correctly classified vessel pixels to the total number of vessel pixels in the ground truth image is termed as Se while the proportion of counted non-vessel pixels to the total number of non-vessel pixels are called as Sp. The PPV is the fraction of pixels properly characterized as vessel pixels that are true vessel pixels. The NPV is the percentage of pixels ordered as non-vessel that are 
calculated appropriately. The FDR is the expected amount of false positives between all substantial assumptions. The MCC is used as a measure of the rate of binary classifications. The JI neglects the true negatives and link the true positives to the amount of sets that belong to the identical class. The DC is defined as the harmonic mean of PPV and Se. The accuracy is the amount of competence to categorize the quantity of conformism of the segmented image to the manually segmented image [40], [41].

We compute the performance matrices for every single image of both the databases by using the formulation presented in Table 1 and then the values are averaged to find a particular performance measure. The Table 2 and 3 illustrates the results. Among the nine performance matrices, Se, Sp and ACC are taken as measures of procedure's presentation to compare the anticipated technique with other existing procedures. The Table 4 represents the comparison of proposed method with different supervised and unsupervised methods given by Mendonca et al. [1] and Farokhain et al. [2], Soares et al. [3], You et al. [4], Marin et al. [5], Fraz et al. [6], Vega et al. [7], Roychowdhury et al. [8], Tan et al. [9], Aslani et al. [11], Zhao et al. [13], Fraz et al. [14], Fraz et al. [15], Zhang et al. [16], Cinsdikici et al. [17], AI-Rawi et al. [18], Azzopardi et al. [19], Budai et al. [20], Odstrcilik et al. [21], Mapayi et al. [22], Panda et al. [23], Roychowdhury et al. [24], AI-Diri et al. [25], Tan et al. [26], Martinez-Perez et al. [28] for DRIVE database. The Table 5 shows the comparison of presented method in terms of Se, Sp and ACC with the published method reported by Fraz et al. [6], Azzopardi et al. [19] for CHASE_DB1 database. The segmentation performances of Marin et al. [5], Azzopardi et al. [19], Odstrcilik et al. [21], Budai et al. [20], Fraz et al. [14], Vega et al. [7], Cinsdikici et al. [17], Fraz et al. [15], Tan et al. [9], Aslani et al. [11], AI-Diri et al. [25], Tan et al. [26], Martinez-Perez et al. [28], Mendonca et al. [1] and Farokhain et al. [2] obtained from the original literature. The segmentation performances of Zhang et al. [16], Soares et al. [3], You et al. [4] are taken from Zhao et al. [13] whereas Fraz et al. [6] and Roychowdhury et al. [8] are taken from Azzopardi et al. [19]. When the proposed method is compared with other existing methods, we can see that it can extracts the retinal vasculature more correctly than many of the existing algorithm. The Fig. 5 depicts the comparison of image DRIVE database with other existing methods. The proposed method is simple and easier to implement. The existing method shows their best performance in detection of thick vessels but unable to give a better result in case of thin vessel detection. So the proposed method also performs better in extraction of thin vessels by giving an average value of Se, Sp and ACC of 0.786, 0.961 and 0.953 for DRIVE database and 0.773, 0.979 and 0.944 for CHASE_DB1 database. Figure 6 depicts extraction of thin vessels in selected sub-region and Table 6 represents the values of different performance metrics in selected sub region. 
Table 1: Performance metrics for retinal blood vessel extraction

\begin{tabular}{|c|c|}
\hline Performance matrices & Expression \\
\hline Se & $\frac{\text { TP }}{\mathrm{TP}+\mathrm{FN}}$ \\
\hline $\mathrm{Sp}$ & $\frac{\mathrm{TN}}{\mathrm{TN}+\mathrm{FP}}$ \\
\hline $\mathrm{PPV}$ & $\frac{\mathrm{TP}}{\mathrm{TP}+\mathrm{FP}}$ \\
\hline $\mathrm{NPV}$ & $\frac{\mathrm{TN}}{\mathrm{TN}+\mathrm{FN}}$ \\
\hline FDR & $\frac{\mathrm{FP}}{\mathrm{FP}+\mathrm{TN}}$ \\
\hline $\mathrm{MCC}$ & $\mathrm{TP} \times \mathrm{TN}-\mathrm{FP} \times \mathrm{FN} / \sqrt{(\mathrm{TP}+\mathrm{FP})(\mathrm{TP}+\mathrm{FN})(\mathrm{TN}+\mathrm{FP})(\mathrm{TN}+\mathrm{FN})}$ \\
\hline JI & $\frac{\mathrm{TP}}{\mathrm{TP}+\mathrm{FP}+\mathrm{FN}}$ \\
\hline DC & $\frac{2 \mathrm{TP}}{2 \mathrm{TP}+\mathrm{FP}+\mathrm{FN}}$ \\
\hline ACC & $\frac{\mathrm{TP}+\mathrm{TN}}{\mathrm{TP}+\mathrm{FN}+\mathrm{TN}+\mathrm{FP}}$ \\
\hline
\end{tabular}


(a)
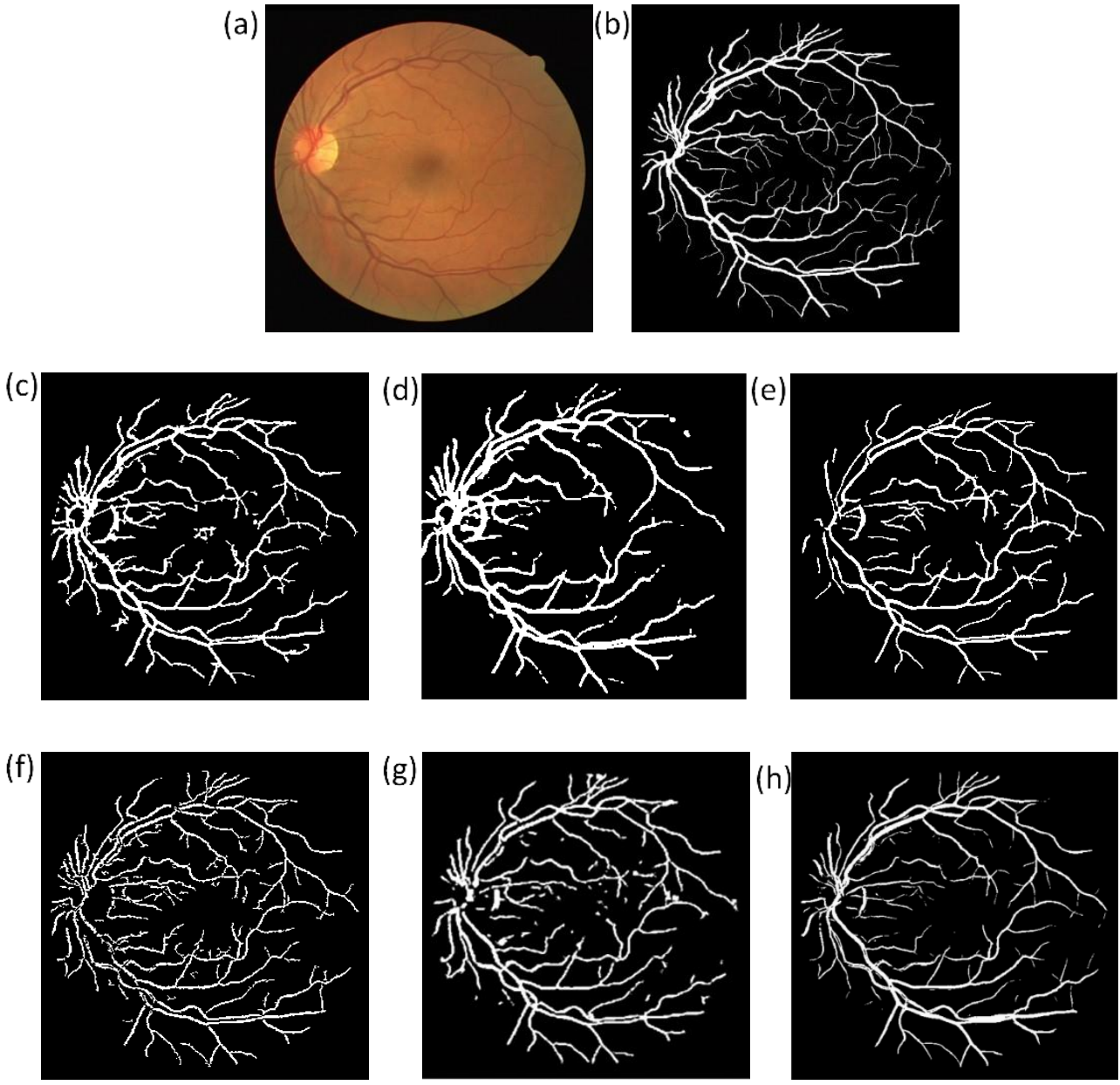

(i)

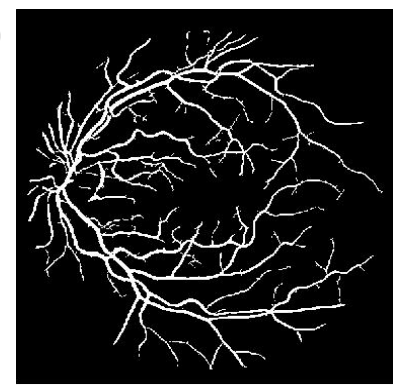

Fig. 5 Segmentation results of first retinal image from the DRIVE database using different methods (a) Original image, (b) ground truth image of the first observer, (c) Cinsdikici et al. [17], (d) AI Rawi et al. [18], (e) Zhang et al. [16], (f) Mapayi et al. [22], (g) Panda et al. [23], (h) Soares et al. [3], and (i) Proposed method. 


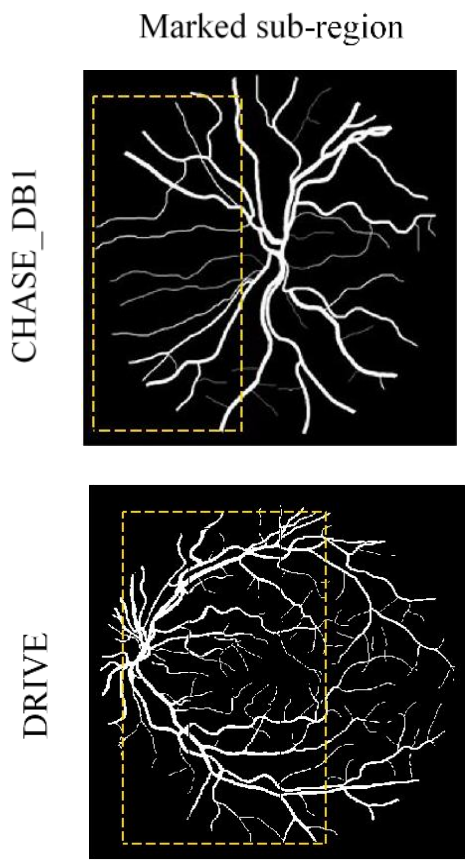

(a)
Ground truth Images
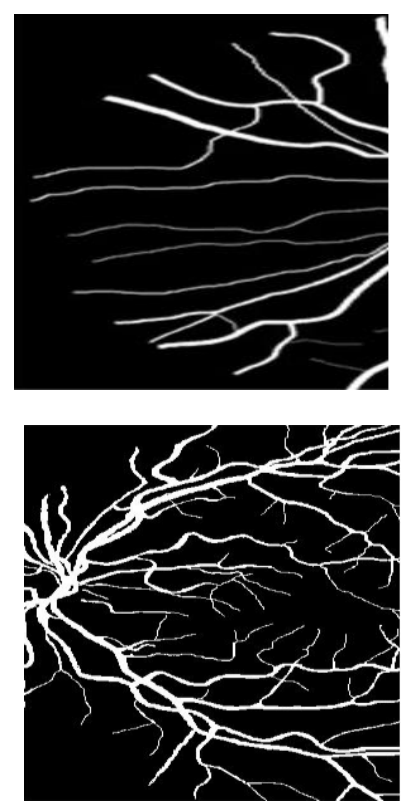

(b)
Segmented Images
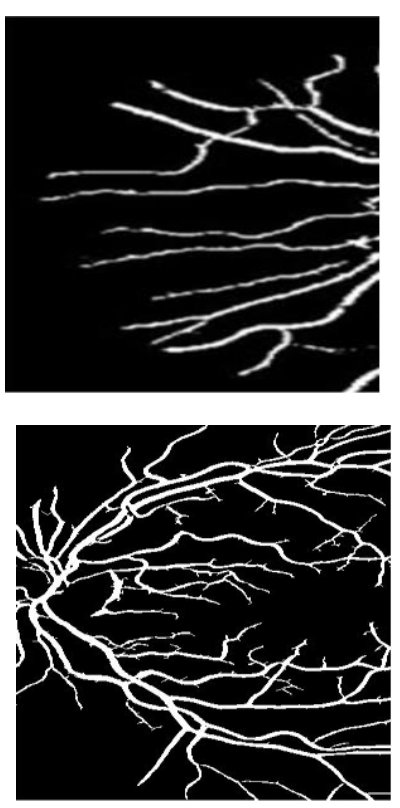

(c)

Fig. 6 Segmentation results of DRIVE, STARE and CHASE_DB1 databases in selected sub-region (a) original images, (b) segmented images

Table 2: Performance evaluation on DRIVE database

\begin{tabular}{|c|c|c|c|c|c|c|c|c|c|}
\hline Image & Se & Sp & PPV & NPV & FDR & MCC & JI & DC & ACC \\
\hline 1 & 0.744 & 0.98 & 0.784 & 0.975 & 0.215 & 0.742 & 0.618 & 0.764 & 0.97 \\
\hline 2 & 0.784 & 0.984 & 0.836 & 0.966 & 0.163 & 0.739 & 0.613 & 0.77 & 0.955 \\
\hline 3 & 0.705 & 0.967 & 0.782 & 0.967 & 0.097 & 0.67 & 0.842 & 0.783 & 0.941 \\
\hline 4 & 0.758 & 0.986 & 0.912 & 0.956 & 0.187 & 0.647 & 0.594 & 0.862 & 0.947 \\
\hline 5 & 0.744 & 0.977 & 0.849 & 0.963 & 0.25 & 0.666 & 0.53 & 0.792 & 0.949 \\
\hline 6 & 0.705 & 0.985 & 0.855 & 0.954 & 0.194 & 0.649 & 0.85 & 0.866 & 0.945 \\
\hline 7 & 0.758 & 0.985 & 0.792 & 0.954 & 0.117 & 0.624 & 0.569 & 0.759 & 0.945 \\
\hline 8 & 0.721 & 0.988 & 0.837 & 0.956 & 0.192 & 0.624 & 0.563 & 0.793 & 0.958 \\
\hline 9 & 0.744 & 0.987 & 0.791 & 0.96 & 0.108 & 0.632 & 0.575 & 0.744 & 0.951 \\
\hline 10 & 0.79 & 0.985 & 0.783 & 0.964 & 0.216 & 0.656 & 0.607 & 0.79 & 0.952 \\
\hline 11 & 0.765 & 0.981 & 0.893 & 0.958 & 0.144 & 0.625 & 0.578 & 0.747 & 0.944 \\
\hline 12 & 0.695 & 0.985 & 0.799 & 0.96 & 0.107 & 0.646 & 0.795 & 0.762 & 0.95 \\
\hline 13 & 0.774 & 0.984 & 0.798 & 0.955 & 0.111 & 0.649 & 0.802 & 0.798 & 0.944 \\
\hline 14 & 0.789 & 0.99 & 0.844 & 0.964 & 0.155 & 0.685 & 0.831 & 0.714 & 0.958 \\
\hline 15 & 0.79 & 0.964 & 0.792 & 0.977 & 0.107 & 0.649 & 0.709 & 0.874 & 0.951 \\
\hline 16 & 0.798 & 0.977 & 0.898 & 0.97 & 0.141 & 0.701 & 0.571 & 0.729 & 0.953 \\
\hline 17 & 0.715 & 0.984 & 0.946 & 0.965 & 0.158 & 0.698 & 0.751 & 0.81 & 0.958 \\
\hline 18 & 0.754 & 0.972 & 0.799 & 0.978 & 0.295 & 0.704 & 0.673 & 0.798 & 0.955 \\
\hline 19 & 0.798 & 0.977 & 0.854 & 0.977 & 0.099 & 0.726 & 0.699 & 0.762 & 0.957 \\
\hline 20 & 0.782 & 0.978 & 0.831 & 0.976 & 0.161 & 0.704 & 0.669 & 0.755 & 0.96 \\
\hline Average & $\mathbf{0 . 7 5 6}$ & $\mathbf{0 . 9 8 1}$ & $\mathbf{0 . 8 3 7}$ & $\mathbf{0 . 9 6 5}$ & $\mathbf{0 . 1 6}$ & $\mathbf{0 . 6 7 2}$ & $\mathbf{0 . 6 9 5}$ & $\mathbf{0 . 7 8 4}$ & $\mathbf{0 . 9 5 2}$ \\
\hline
\end{tabular}


Table 3: Performance evaluation on CHASE DB1 database

\begin{tabular}{|c|c|c|c|c|c|c|c|c|c|}
\hline Image & Se & Sp & PPV & NPV & FDR & MCC & JI & DC & ACC \\
\hline 1 & 0.856 & 0.966 & 0.776 & 0.97 & 0.223 & 0.56 & 0.719 & 0.797 & 0.962 \\
\hline 2 & 0.754 & 0.964 & 0.874 & 0.96 & 0.125 & 0.535 & 0.59 & 0.761 & 0.95 \\
\hline 3 & 0.736 & 0.974 & 0.778 & 0.969 & 0.221 & 0.629 & 0.789 & 0.757 & 0.948 \\
\hline 4 & 0.751 & 0.975 & 0.698 & 0.97 & 0.101 & 0.647 & 0.708 & 0.773 & 0.95 \\
\hline 5 & 0.71 & 0.983 & 0.795 & 0.964 & 0.154 & 0.632 & 0.784 & 0.773 & 0.951 \\
\hline 6 & 0.692 & 0.986 & 0.714 & 0.959 & 0.185 & 0.566 & 0.611 & 0.883 & 0.95 \\
\hline 7 & 0.699 & 0.982 & 0.756 & 0.961 & 0.273 & 0.897 & 0.645 & 0.776 & 0.955 \\
\hline 8 & 0.779 & 0.986 & 0.764 & 0.971 & 0.155 & 0.73 & 0.79 & 0.861 & 0.951 \\
\hline 9 & 0.716 & 0.968 & 0.705 & 0.979 & 0.089 & 0.734 & 0.587 & 0.799 & 0.951 \\
\hline 10 & 0.835 & 0.98 & 0.75 & 0.958 & 0.149 & 0.414 & 0.777 & 0.834 & 0.941 \\
\hline 11 & 0.779 & 0.96 & 0.79 & 0.982 & 0.109 & 0.549 & 0.698 & 0.699 & 0.945 \\
\hline 12 & 0.775 & 0.979 & 0.748 & 0.964 & 0.231 & 0.569 & 0.522 & 0.774 & 0.947 \\
\hline 13 & 0.749 & 0.976 & 0.593 & 0.968 & 0.106 & 0.568 & 0.583 & 0.793 & 0.959 \\
\hline 14 & 0.744 & 0.978 & 0.776 & 0.973 & 0.213 & 0.741 & 0.797 & 0.794 & 0.955 \\
\hline Average & $\mathbf{0 . 7 7}$ & $\mathbf{0 . 9 7}$ & $\mathbf{0 . 7 5}$ & $\mathbf{0 . 9 8}$ & $\mathbf{0 . 1 2}$ & $\mathbf{0 . 6 3}$ & $\mathbf{0 . 6 9}$ & $\mathbf{0 . 8}$ & $\mathbf{0 . 9 5}$ \\
\hline
\end{tabular}

Table 4: Segmentation performance comparisons of different methods on the DRIVE database

\begin{tabular}{|c|c|c|c|c|}
\hline \multirow{4}{*}{} & Method & Se & Sp & Acc \\
\cline { 2 - 5 } & Soares et al. [3] & - & - & 0.946 \\
\cline { 2 - 5 } & You et al. [4] & 0.741 & 0.975 & 0.943 \\
\cline { 2 - 5 } & Marin et al. [5] & 0.706 & 0.98 & 0.945 \\
\cline { 2 - 5 } & Fraz et al. [6] & 0.74 & 0.98 & 0.948 \\
\cline { 2 - 5 } & Vega et al. [7] & 0.744 & 0.96 & 0.941 \\
\cline { 2 - 5 } & Roychowdhury et al. [8] & 0.724 & 0.983 & 0.951 \\
\cline { 2 - 5 } & Tan et al. [9] & - & - & 0.926 \\
\hline Aslani et al. [11] & 0.754 & 0.98 & 0.951 \\
\hline Mendonca et al. [1] & 0.734 & 0.976 & 0.945 \\
\hline Farokhian et al. [2] & 0.693 & 0.979 & 0.939 \\
\hline Zhao et al. [13] & 0.735 & 0.978 & 0.947 \\
\cline { 2 - 5 } & Fraz et al. [14] & 0.715 & 0.976 & 0.943 \\
\cline { 2 - 5 } & Fraz et al. [15] & 0.73 & 0.972 & 0.942 \\
\cline { 2 - 5 } & Zhang et al. [16] & 0.712 & 0.972 & 0.938 \\
\cline { 2 - 5 } & Cinsdikici et al. [17] & - & - & 0.929 \\
\cline { 2 - 5 } & AI-Rawi et al.[18] & - & - & 0.942 \\
\cline { 2 - 5 } & Azzopardi et al. [19] & 0.765 & 0.97 & 0.944 \\
\cline { 2 - 5 } & Budai et al. [20] & 0.644 & 0.987 & 0.957 \\
\cline { 2 - 5 } & Odstrcilik et al. [21] & 0.706 & 0.969 & 0.934 \\
\cline { 2 - 5 } & Mapayi et al. [22] & 0.731 & 0.972 & 0.951 \\
\cline { 2 - 5 } & Panda et al. [23] & 0.733 & 0.975 & 0.953 \\
\hline & Tan et al. [26] & - & - & 0.93 \\
\hline
\end{tabular}




\begin{tabular}{|c|c|c|c|c|}
\hline & Martinez-Perez et al. [28] & 0.724 & 0.933 & 0.934 \\
\cline { 2 - 5 } & Proposed Method & $\mathbf{0 . 7 5 6}$ & $\mathbf{0 . 9 8 1}$ & $\mathbf{0 . 9 5 2}$ \\
\hline
\end{tabular}

Table 5: Segmentation performance comparisons of different methods on the CHASE DB1 database

\begin{tabular}{|l|c|c|c|}
\hline Method & Se & Sp & Acc \\
\hline Fraz et al. [6] & 0.722 & 0.971 & 0.946 \\
\hline Azzopardi et al. [19] & 0.758 & 0.958 & 0.938 \\
\hline Proposed method & $\mathbf{0 . 7 7 4}$ & $\mathbf{0 . 9 7 4}$ & $\mathbf{0 . 9 5 1}$ \\
\hline
\end{tabular}

Table 6: Performance evaluation on sub-region

\begin{tabular}{|c|c|c|}
\hline Performance matrices & DRIVE & CHASE_DB1 \\
\hline Se & 0.786 & 0.773 \\
\hline Sp & 0.961 & 0.979 \\
\hline PPV & 0.776 & 0.813 \\
\hline NPV & 0.964 & 0.958 \\
\hline FDR & 0.223 & 0.186 \\
\hline MCC & 0.744 & 0.71 \\
\hline JI & 0.64 & 0.683 \\
\hline DC & 0.781 & 0.736 \\
\hline ACC & 0.953 & 0.944 \\
\hline
\end{tabular}

\section{Conclusion}

This paper presents an unsupervised method for extraction of blood vessels from fundus images. This process extracts vessel pixels from a vessel enhanced image from the green plane from each fundus image. The algorithm starts with vessel enhancement using CLAHE followed by extraction of features by Gabor filtering. It further enhances the extracted featured image with the application of Hessian based enhancement filter. Further, the total vasculature is extracted from background by using K-mean clustering and a final vessels segmented image is obtained using a morphological cleaning operation. It maintains consistency in giving accuracy for all the images of both the databases. This method outperforms many of the existing algorithm by giving an average value of Se, Sp and ACC of $0.756,0.981$ and 0.952 for DRIVE database and 0.774, 0.974 and 0.951 for CHASE_DB1 database. Besides that, it also performs better in detecting thin vessels by giving average accuracies of 0.953 and 0.944 for DRIVE and CHASE_DB1 databases, respectively. The main advantage of the proposed method is that it is simple, easier to implement and do not require training as it is unsupervised in nature.

\section{References}

[1] A. M. Mendonca and A. Campilho, "Segmentation of retinal blood vessels by combining the detection of centerlines and morphological reconstruction," IEEE Transactions on Medical Imaging, vol. 25, no. 9, pp. 1200-1213, Sep. 2006.

[2] F. Farokhian, C. Yang, H. Demirel, S. Wu, and I. Beheshti, "Automatic parameters selection of 
Gabor filters with the imperialism competitive algorithm with application to retinal vessel segmentation," Biocybernetics and Biomedical Engineering, vol. 37, no. 1, pp. 246-254, 2017.

[3] J. V. B. Soares, J. J. G. Leandro, R. M. Cesar, H. F. Jelinek, and M. J. Cree, "Retinal vessel segmentation using the 2-D Gabor wavelet and supervised classification," IEEE Transactions on Medical Imaging, vol. 25, no. 9, pp. 1214-1222, Sep. 2006.

[4] X. You, Q. Peng, Y. Yuan, Y. Cheung, and J. Lei, "Segmentation of retinal blood vessels using the radial projection and semi-supervised approach," Pattern Recognition, vol. 44, no. 10-11, pp. 23142324, Oct. 2011.

[5] D. Marín, A. Aquino, M. E. Gegundez-Arias, and J. M. Bravo, "A New Supervised Method for Blood Vessel Segmentation in Retinal Images by Using Gray-Level and Moment Invariants-Based Features," IEEE Transactions on Medical Imaging, vol. 30, no. 1, pp. 146-158, Jan. 2011.

[6] M. M. Fraz et al., "An Ensemble Classification-Based Approach Applied to Retinal Blood Vessel Segmentation," IEEE Transactions on Biomedical Engineering, vol. 59, no. 9, pp. 2538-2548, Sep. 2012.

[7] R. Vega, G. Sanchez-Ante, L. E. Falcon-Morales, H. Sossa, and E. Guevara, "Retinal vessel extraction using Lattice Neural Networks with dendritic processing," Computers in Biology and Medicine, vol. 58, pp. 20-30, Mar. 2015.

[8] S. Roychowdhury, D. Koozekanani, and K. Parhi, "Blood Vessel Segmentation of Fundus Images by Major Vessel Extraction and Sub-Image Classification," IEEE Journal of Biomedical and Health Informatics, pp. 1-1, 2014.

[9] J. H. Tan et al., "Automated segmentation of exudates, haemorrhages, microaneurysms using single convolutional neural network," Information Sciences, vol. 420, pp. 66-76, Dec. 2017.

[10] J. H. Tan, U. R. Acharya, S. V. Bhandary, K. C. Chua, and S. Sivaprasad, "Segmentation of optic disc, fovea and retinal vasculature using a single convolutional neural network," Journal of Computational Science, Feb. 2017.

[11] S. Aslani and H. Sarnel, "A new supervised retinal vessel segmentation method based on robust hybrid features," Biomedical Signal Processing and Control, vol. 30, pp. 1-12, Sep. 2016.

[12] L. Câmara Neto, G. L. B. Ramalho, J. F. S. Rocha Neto, R. M. S. Veras, and F. N. S. Medeiros, "An unsupervised coarse-to-fine algorithm for blood vessel segmentation in fundus images," Expert Systems with Applications, vol. 78, pp. 182-192, Jul. 2017.

[13] Y. Qian Zhao, X. Hong Wang, X. Fang Wang, and F. Y. Shih, "Retinal vessels segmentation based on level set and region growing," Pattern Recognition, vol. 47, no. 7, pp. 2437-2446, Jul. 2014.

[14] M. M. Fraz et al., "An approach to localize the retinal blood vessels using bit planes and centerline detection," Computer Methods and Programs in Biomedicine, vol. 108, no. 2, pp. 600-616, Nov. 2012.

[15] M. M. Fraz, A. Basit, and S. A. Barman, "Application of Morphological Bit Planes in Retinal Blood Vessel Extraction,” Journal of Digital Imaging, vol. 26, no. 2, pp. 274-286, Apr. 2013.

[16] B. Zhang, L. Zhang, L. Zhang, and F. Karray, "Retinal vessel extraction by matched filter with firstorder derivative of Gaussian," Computers in Biology and Medicine, vol. 40, no. 4, pp. 438-445, Apr. 2010.

[17] M. G. Cinsdikici and D. Aydin, "Detection of blood vessels in ophthalmoscope images using MF/ant (matched filter/ant colony) algorithm.," Computer methods and programs in biomedicine, vol. 96, no. 2, pp. 85-95, Nov. 2009.

[18] M. Al-Rawi and H. Karajeh, "Genetic algorithm matched filter optimization for automated detection of blood vessels from digital retinal images," Computer Methods and Programs in Biomedicine, vol. 87, no. 3, pp. 248-253, Sep. 2007.

[19] G. Azzopardi, N. Strisciuglio, M. Vento, and N. Petkov, "Trainable COSFIRE filters for vessel 
delineation with application to retinal images," Medical Image Analysis, vol. 19, no. 1, pp. 46-57, Jan. 2015.

[20] A. Budai, R. Bock, A. Maier, J. Hornegger, and G. Michelson, "Robust Vessel Segmentation in Fundus Images," International Journal of Biomedical Imaging, vol. 2013, pp. 1-11, 2013.

[21] R. Kolar et al., "Retinal vessel segmentation by improved matched filtering: evaluation on a new high-resolution fundus image database," IET Image Processing, vol. 7, no. 4, pp. 373-383, Jun. 2013.

[22] T. Mapayi, S. Viriri, and J.-R. Tapamo, "Adaptive Thresholding Technique for Retinal Vessel Segmentation Based on GLCM-Energy Information," Computational and Mathematical Methods in Medicine, vol. 2015, pp. 1-11, 2015.

[23] R. Panda, N. B. Puhan, and G. Panda, "New Binary Hausdorff Symmetry measure based seeded region growing for retinal vessel segmentation," Biocybernetics and Biomedical Engineering, vol. 36, no. 1, pp. 119-129, 2016.

[24] S. Roychowdhury, D. D. Koozekanani, and K. K. Parhi, "Iterative Vessel Segmentation of Fundus Images," IEEE Transactions on Biomedical Engineering, vol. 62, no. 7, pp. 1738-1749, Jul. 2015.

[25] B. Al-Diri, A. Hunter, and D. Steel, "An Active Contour Model for Segmenting and Measuring Retinal Vessels," IEEE Transactions on Medical Imaging, vol. 28, no. 9, pp. 1488-1497, Sep. 2009.

[26] J. H. Tan, U. R. Acharya, K. C. Chua, C. Cheng, and A. Laude, "Automated extraction of retinal vasculature.," Medical physics, vol. 43, no. 5, p. 2311, May 2016.

[27] O. Chutatape, Liu Zheng, and S. M. Krishnan, "Retinal blood vessel detection and tracking by matched Gaussian and Kalman filters," in Proceedings of the 20th Annual International Conference of the IEEE Engineering in Medicine and Biology Society. Vol.20 Biomedical Engineering Towards the Year 2000 and Beyond (Cat. No.98CH36286), vol. 6, pp. 3144-3149.

[28] M. E. Martinez-Perez, A. D. Hughes, S. A. Thom, A. A. Bharath, and K. H. Parker, "Segmentation of blood vessels from red-free and fluorescein retinal images," Medical Image Analysis, vol. 11, no. 1, pp. 47-61, Feb. 2007.

[29] J. Dash and N. Bhoi, "Detection of retinal blood vessels from ophthalmoscope images using morphological approach," ELCVIA Electronic Letters on Computer Vision and Image Analysis, vol. 16, no. 1, p. 1, Feb. 2017.

[30] K. Zuiderveld, "Contrast Limited Adaptive Histogram Equalization," in Graphics Gems, Elsevier, 1994, pp. 474-485.

[31] T. Walter, P. Massin, A. Erginay, R. Ordonez, C. Jeulin, and J.-C. Klein, “Automatic detection of microaneurysms in color fundus images," Medical Image Analysis, vol. 11, no. 6, pp. 555-566, Dec. 2007.

[32] M. Haghighat, S. Zonouz, and M. Abdel-Mottaleb, "CloudID: Trustworthy cloud-based and crossenterprise biometric identification," Expert Systems with Applications, vol. 42, no. 21, pp. 79057916, Nov. 2015.

[33] J.-K. Kamarainen, "Gabor features in image analysis," in 2012 3rd International Conference on Image Processing Theory, Tools and Applications (IPTA), 2012, pp. 13-14.

[34] S.-F. Yang and C.-H. Cheng, "Fast computation of Hessian-based enhancement filters for medical images," Computer Methods and Programs in Biomedicine, vol. 116, no. 3, pp. 215-225, Oct. 2014.

[35] N. Dhanachandra, K. Manglem, and Y. J. Chanu, "Image Segmentation Using K -means Clustering Algorithm and Subtractive Clustering Algorithm," Procedia Computer Science, vol. 54, pp. 764-771, 2015.

[36] K. Michielsen and H. De Raedt, Morphological image analysis, vol. 132, no. 1-2. 2000.

[37] J. Staal, M. D. Abramoff, M. Niemeijer, M. A. Viergever, and B. van Ginneken, "Ridge-Based Vessel Segmentation in Color Images of the Retina," IEEE Transactions on Medical Imaging, vol. 23, no. 4, pp. 501-509, Apr. 2004. 
[38] C. G. Owen et al., "Measuring retinal vessel tortuosity in 10-year-old children: validation of the Computer-Assisted Image Analysis of the Retina (CAIAR) program.," Investigative ophthalmology \& visual science, vol. 50, no. 5, pp. 2004-10, May 2009.

[39] J. Dash and N. Bhoi, "A method for blood vessel segmentation in retinal images using morphological reconstruction," in 2016 International Conference on Computer, Electrical \& Communication Engineering (ICCECE), 2016, pp. 1-5.

[40] J. Dash and N. Bhoi, "A thresholding based technique to extract retinal blood vessels from fundus images," Future Computing and Informatics Journal, vol. 2, no. 2, pp. 103-109, Dec. 2017.

[41] J. Dash and N. Bhoi, "An Unsupervised Approach for Extraction of Blood Vessels from Fundus Images,” Journal of Digital Imaging, Apr. 2018. 